\title{
Asthma und Vitamin D
}

\author{
Autor: Jörg Spitz
}

\section{ZUSAMMENFASSUNG}

Deutschlandweit sind über 3 Millionen Erwachsene von Asthma bronchiale und über 12 Millionen von allergischer Rhinitis betroffen. Studien belegen, dass Vitamin D eng mit der Regulation des Immunsystems verbunden ist. Die gezielte Beseitigung eines Vitamin-D-Mangels führt daher zu deutlich positiven Auswirkungen bei diesen Erkrankungen sowie bei Infektionskrankheiten und Autoimmunerkrankungen. Die Voraussetzung für eine erfolgreiche Vorbeugung und Behandlung ist eine tägliche Dosis des Sonnenhormons, die den Vitamin-D-Spiegel ganzjährig in einem Bereich von 40-60 ng/ml hält.

\section{Schlüsselwörter}

Asthma bronchiale, allergische Rhinitis, Vitamin D, Vitamin-

D-Dosis, Vitamin-D-Supplementation, Immunmodulation

\section{ABSTRACT}

In Germany, more than 3 million adults are suffering from bronchial asthma, and more than 12 million from allergic rhinitis. Studies show, that vitamin $\mathrm{D}$ is closely linked to the regulation of the immune system. Therefore, the selective elimination of a vitamin D deficiency has positive effects in the case of these diseases as well as in the case of infectious diseases and autoimmune diseases. The prerequisite for a successful prevention and treatment is a daily dose of the sun hormone, which keeps the level of vitamin $D$ in a range of $40-60 \mathrm{ng} / \mathrm{ml}$ during the whole year.

\section{Keywords}

Bronchial asthma, allergic rhinitis, vitamin D, vitamin D dose, vitamin D supplementation, immunomodulation

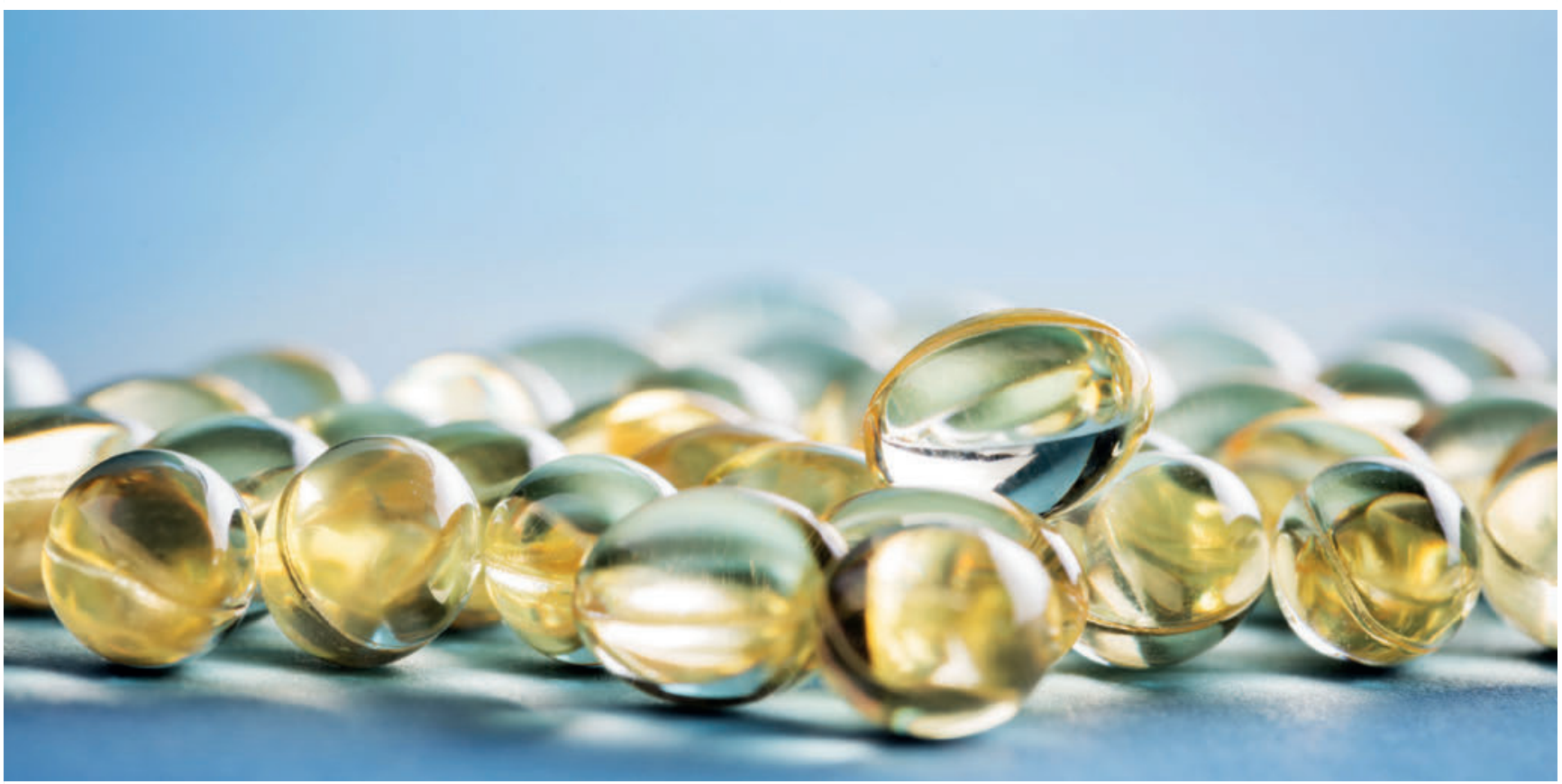

- Abb. 1 Zur Substitution von Vitamin D eignen sich alle Präparate in ausreichender Dosierung, wenn sie zu einer fettreichen Mahlzeit eingenommen werden, unabhängig davon, ob sie das fettlösliche Hormon in Öl gelöst enthalten. @ Photocreo Bednarek/Adobe Stock

Die nachstehenden Ausführungen zur Epidemiologie beruhen auf bundesweiten, repräsentativen Untersuchungen des Robert Koch Institutes, die im Auftrag der Bundesregierung durchgeführt und publiziert wurden. Asthma bronchiale und allergische Rhinitis sind Erkrankungen mit hoher Krankheitslast, deren Symptome zu einer erheblichen Beeinträchtigung der Lebensqualität führen können. Deutschlandweit sind mehr als 3 Millionen Erwachsene von Asthma bronchiale betroffen; mehr als 12 Millionen Erwachsene berichten, unter einer allergischen Rhinitis zu leiden. Frauen sind häufiger von Asthma bronchiale und allergischer Rhinitis betroffen als Männer. Im Zehnjahreszeitraum zwischen BGS98 (1997-1999) und DEGS1 (20082011) hat sich der Anteil der Asthmatiker erhöht [15].

Bei Kindern sieht es nicht viel besser aus als bei Erwachsenen: Zwischen den beiden repräsentativen Untersuchungen an Kindern von 2006 und 2012 ist der Anteil der Asthmatiker statistisch signifikant angestiegen, v. a. bei kleinen Kindern bis 6 Jahre. Aktuell sind 0,5 Millionen Kinder und 
Jugendliche betroffen. Jedes 5. Kind mit Asthma hat Fehlzeiten in Kita oder Schule aufgrund seiner Erkrankung, im Durchschnitt 1 von 38 Schulwochen im Jahr [6].

Ein Hinweis auf eine Verbindung zwischen diesen Erkrankungen und dem bevölkerungsweiten Vitamin-D-Mangel findet sich jedoch in keiner der vorstehenden repräsentativen Publikationen. Das ist umso erstaunlicher, als die Verbindung von Vitamin D und dem Immunsystem einen von der Europäischen Behörde für Lebensmittelsicherheit (EFSA) anerkannten „Health Claim“ darstellt [4]. Grund genug, mit den nachfolgenden Fakten gezielte Aufklärungsarbeit zu leisten.

\section{Paradigmenwechsel bei Vitamin D}

Seit meinem ersten Buch zur Darstellung der erweiterten Eigenschaften von Vitamin D vor rund zehn Jahren [17] wurden zwar zahlreiche weitere Bücher auch von anderen Autoren sowie Tausende wissenschaftlicher Publikationen zu diesem Thema veröffentlicht. Das Wissen um die Eigenschaften und Wirkweise von Vitamin D ist jedoch noch lange kein Allgemeingut. Das trifft auch und insbesondere immer noch auf die medizinische Praxis zu. Angesichts des mehr als umfangreichen Materials werden die grundlegenden Eigenschaften des „Sonnenhormons“ hier nur schlaglichtartig beleuchtet. Bei der Erforschung der Eigenschaften von Vitamin D gab es gleich mehrere Paradigmenwechsel [17]:

- Vitamin D ist gar kein Vitamin, das wir essen müssen, wie die übrigen Vitamine, sondern ein Prohormon, das in der Haut mithilfe der UVB-Strahlung der Sonne gebildet wird. Die Haut wird damit zu einer Drüse und aus dem Vitamin ein Sonnenhormon.

- Vitamin D wird nicht nur für den Knochenstoffwechsel, sondern für praktisch alle Organe und Zellen im Körper, die über Vitamin-D-Rezeptoren verfügen, benötigt.

- Zu den vielfältigen Aufgaben des Sonnenhormons gehört u. a. die epigenetische Steuerung von rund 2000 Genen. So beeinflusst Vitamin D sowohl das angeborene als auch das erworbene Immunsystem und ist in der Lage, körpereigene Antibiotika herzustellen.

- Nicht nur Säuglinge haben einen Mangel an Vitamin D, sondern praktisch die gesamte Bevölkerung. Der Grund hierfür ist der grundlegend veränderte Lebensstil.

- Ein Mangel an Vitamin D beeinflusst nicht nur die Entstehung und den Verlauf von Rachitis und Osteoporose, sondern nahezu aller chronischen Krankheiten.

Bei der Beschäftigung mit dem Sonnenhormon wird immer deutlicher, dass die Bedeutung von Vitamin D für das Immunsystem - und damit für alle Autoimmunerkrankungen - weitaus größer ist, als ursprünglich gedacht. Wir haben dem Thema „Autoimmunerkrankungen“ daher den Kongress für menschliche Medizin 2018 gewidmet und stellen die videodokumentierten Vorträge als weiterführende Informationen auf der Website des Kongresses [9] und der Akademie für menschliche Medizin zur Verfügung. Speziell in Bezug auf allergische Atemwegserkrankungen gibt es inzwischen mehrere aktuelle Publikationen zur Bedeutung von Vitamin D, die nachfolgend näher vorgestellt werden.

Liste der mit Vitamin D assoziierten Eigenschaften und wesentlichen Wirkungen auf unseren Körper (unvollständiger Auszug, [17][19]):

- 70-90\% Vitamin-D-Mangel in der Bevölkerung (darunter auch besonders vulnerable Gruppen wie Schwangere und Kinder, aber auch die Mehrzahl chronisch kranker Menschen)

- fördert die Fertilität

- schützt den Schwangerschaftsverlauf

- fördert das angeborene und das erworbene Immunsystem

- reduziert das Risiko für Alzheimer, Demenz

- schützt die Nervenzellen (z. B. vor MS)

- bremst die Krebsentwicklung

- verhindert die Entstehung von Metastasen

- senkt den Blutdruck

- verbessert das Überleben von Patienten mit Koronarer Herzkrankheit (KHK)

- reduziert das Risiko für Diabetes

- kräftigt die Muskulatur, verzögert Pflegebedürftigkeit im Alter

\section{Vitamin D und das Immunsystem}

Bei der Herausgabe meines ersten Buches vor zehn Jahren waren die klinischen Erfahrungen noch begrenzt und die Mehrzahl der damals etwa 40 wissenschaftlichen Artikel in der Datenbank pubmed überwiegend experimenteller Natur. Heute finden sich zahlreiche Beobachtungsstudien zum Thema „Sonnenhormon und Immunsystem“, ebenso wie therapeutische Studien zur gezielten Beeinflussung des Immunsystems durch Vitamin D. Konsequenterweise gibt es in diesem Jahr auch eine Neuauflage meines Buches unter dem Titel „Vitamin-D-Mangel: die unterschätzte Gefahr“ [18]. Vitamin-D-Skeptiker führen gerne das Argument an, dass Vitamin D das Immunsystem einseitig herunterreguliert und schwächt. Die Wirklichkeit ist jedoch viel komplexer und die Einflussnahme von Vitamin D wird zu Recht auch als „Immunmodulation“ bezeichnet. Eine aktuelle Übersichtsarbeit aus dem Jahr 2017 [3] bezeichnet Vitamin D gar als „Überlebensmolekül“, das auf unterschiedlichen Wegen das Immungeschehen steuert. Demnach werden durch Modulation des Immunsystems zum einen Entzündungsreaktionen infolge von zellulärem Stress gehemmt, zum anderen spezielle Immunzellen zur Unterstützung von Reparaturprozessen gefördert.

Skeptiker, die gerne die Beweiskraft vergleichender epidemiologischer Untersuchungen an Patienten und Kontroll- 


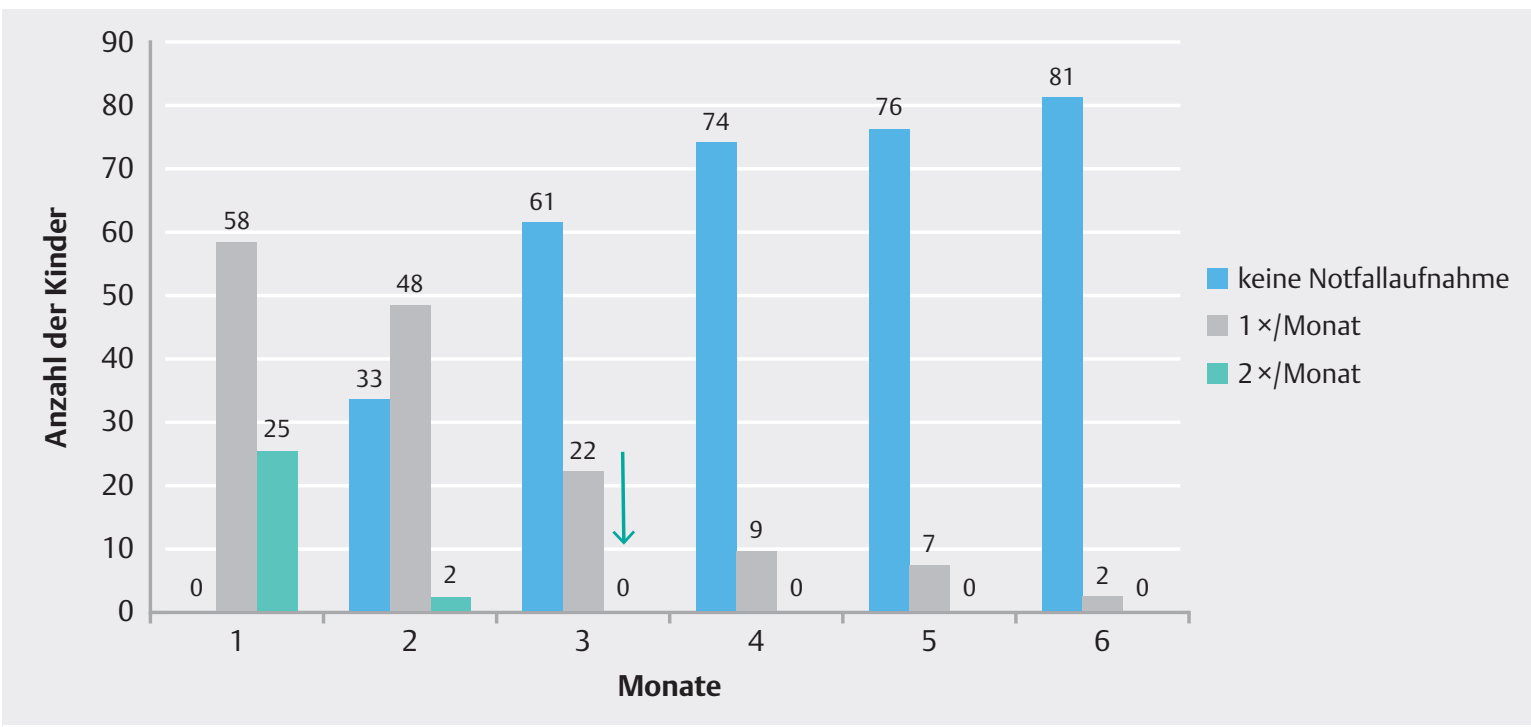

- Abb. 2 Der Rückgang der Häufigkeit von Notfallaufnahmen bei Kindern pro Monat als Effekt einer konsequenten Supplementation mit Vitamin D ist eindrucksvoll [10].

personen anzweifeln, seien daran erinnert, dass es parallel zu diesen Beobachtungsstudien eine Fülle von Laboruntersuchungen gibt, die die exakten Wirkungsmechanismen von Vitamin D in den Zellen aufzeigen. Als Beispiel dient die Darstellung der Bedeutung des Botenstoffs Interleukin 32 [12]. Diese Art von biochemischen Untersuchungen sind zwar kompliziert, es ist jedoch gerade für Ärzte empfehlenswert, ihre aussagekräftigen Ergebnisse zu kennen. IL-32 ist ein molekularer Marker des Abwehrnetzwerkes bei Tuberkulose. Dabei induziert IL-32 die von Vitamin D abhängige Produktion der antimikrobiellen Peptide Cathelicidin und DEFB4 und erzeugt damit eine antimikrobielle Aktivität (d. h., eine Wirkung wie bei einem Antibiotikum), die im Laborversuch vom Vorhandensein einer adäquaten Menge von 25-OH Vitamin D abhängig war [12].

Dennoch ist Vitamin D, zumindest bei denen, die seine Bedeutung verstanden haben, inzwischen im therapeutischen Alltag angekommen - auch in Bezug auf die Atemwege. So zeigt eine 2017 erschienene Übersichtsarbeit der Arbeitsgruppe von Martineau die positiven Auswirkungen einer Vitamin-D-Supplementation auf akute Atemwegsinfekte im Rahmen der Asthmaerkrankung. Die Auswertung umfasst 25 evidenzbasierte Studien mit über 10000 Teilnehmern [11]. Die statistische Auswertung aller untersuchten Patienten unter einer Vitamin-D-Supplementation ergab eine bereits beachtliche Risikoreduktion von $12 \%$ für eine akute Erkrankung der Luftwege. Eine Subgruppenanalyse zeigte für Patienten unter einer täglichen oder wöchentlichen Vitamin-D-Gabe sogar noch eine deutlich höhere Risikoreduktion von 19\%. Damit bestätigt diese Auswertung die Beobachtungen von Hollis bezüglich der höheren Effektivität täglicher Vitamin-D-Gaben, auf die ich bereits seit einiger Zeit verweise [7]. Ferner profitierten diejenigen Patienten von Vitamin-D-Gaben am meisten, die einen sehr niedrigen Vitamin-D-Ausgangswert hatten.
Das ist eine Erkenntnis, die in zahlreichen anderen Studien ebenfalls gemacht wurde. In der Meta-Analyse von Martineau betrug die Risikoreduktion $25 \%$ bei einem Ausgangswert von $<10 \mathrm{ng} / \mathrm{ml}$ Vitamin D im Blut (im Vergleich zu $12 \%$ im Gesamtkollektiv).

Was für Erwachsenen gilt, gilt auch für Kinder - zumindest in Bezug auf Vitamin D, Infekte und Asthma. Die Arbeitsgruppe von Krishnan berichtete 2017 über 96 Kinder mit Asthma, von denen $86 \%$ einen Vitamin-D-Mangel hatten, wobei letzterer signifikant mit den Schweregrad und der Kontrollierbarkeit des Asthmas sowie dem Nachweis von eosinophilen (entzündungstypischen) Leukozyten im Blut verbunden war [10] ( Abb. 2). Über ähnliche Erfahrungen berichtete im gleichen Jahr die Arbeitsgruppe von Alansari. Sie fand bei ebenfalls knapp 100 Kindern mit deutlich niedrigen Vitamin-D-Spiegeln bereits drei Monate nach Beginn der Supplementation eine Reduktion der Exazerbationshäufigkeit der Asthmaerkrankung um rund $50 \%$ [1].

Die Arbeitsgruppe von Norlin versorgte 277 Patienten mit 1500 IE Vitamin D pro Tag und registrierte während der anschließenden zwölf Monate Veränderungen in Bezug auf den Einsatz von Antibiotika im Vergleich zum Jahr zuvor. Das Ergebnis kann sich sehen lassen: Eine signifikante Reduktion des durchschnittlichen Antibiotikabedarfs von 20 auf 15 Tage sowie ein Anstieg der Anzahl von Patienten, die keine Antibiotika benötigten von 52 auf 81 Patienten. Ferner fand sich eine deutlich geringere Anzahl der Verschreibungen von Antibiotika [13].

Diesen Berichten von internationalen Untersuchungen entsprechen die Ergebnisse eines Pilotprojektes (d.h., einer orientierenden Untersuchung ohne Placebo, Verblindung und Randomisierung), für das ich bereits vor zehn Jahren drei Lungenfachärzte gewinnen konnte (Dr. 
Hermann Teutemacher, Wuppertal, Dr. Hubert Trötschler, Freiburg und Dr. Andreas Deimling, Schleswig). Sie untersuchten und behandelten innerhalb eines Jahres über 900 Patienten mit chronisch obstruktiven Atemwegserkrankungen in ihren Praxen (in der Regel 3000 IE Vitamin D pro Tag für 70 kg Körpergewicht). Die gemessenen Ausgangswerte für Vitamin D lagen im Mittel bei $16 \mathrm{ng} / \mathrm{ml}$, wobei dieser Mittelwert selbst in den Sommermonaten nicht über $20 \mathrm{ng} / \mathrm{ml}$ anstieg. Bei rund $80 \%$ der Patienten kam es zu einer deutlichen Verbesserung des Allgemeinbefindens, wobei bei der Verlaufsbeobachtung auch zusätzliche positive Effekte neben der Verbesserung der Symptomatik der Atemwege berücksichtigt wurden [14]. Ein Erfahrungsbericht, der leider in den pulmologischen Praxen Deutschlands bislang keine größere Beachtung gefunden hat, wie die Publikationen des Robert Koch Instituts eindrücklich belegen.

Anfang 2018 zeigte die Arbeitsgruppe von Zhang in einem eleganten tierexperimentellen Ansatz die direkte Wirkung von Vitamin D auf das entzündliche Geschehen im Lungengewebe [20]. Nach experimentell induziertem Asthma zeigte das Ausmaß der Infiltration eine deutliche Abhängigkeit von den Vitamin-D-Dosierungen. Hohe Dosierungen waren mit einem geringeren Ausmaß entzündlicher Infiltrationen im Lungengewebe assoziiert.

\section{Vitamin-D-Polymorphismen und Immunsystem}

Neben der Abhängigkeit entzündlicher Atemwegsveränderungen von der Versorgung mit Vitamin D hat sich in den vergangenen Jahren ein weiteres, neues Forschungsgebiet für Vitamin D eröffnet: Gen-Polymorphismen. Diese neuen Untersuchungen zeigen, dass selbst geringfügige Veränderungen der Polymorphismen zu einer Beeinträchtigung der Funktion eines Gens für bestimmte Zellfunktionen führen können. Vitamin D ist an der Steuerung von etwa 2000 Genen beteiligt, daher ist zu erwarten, dass diese Gen-Polymorphismen auch zu entsprechenden Beeinträchtigungen der Vitamin- D-Funktion im Körper führen. In diesem Zusammenhang sind Veränderungen an den Vitamin-D-Rezeptoren (VDR) von besonderem Interesse, da diese Rezeptoren benötigt werden, um die Funktion von Vitamin D in den Zellen auszulösen. Von den mehr als 500 Artikeln, die alleine in der Datenbank pubmed zu diesem Thema zu finden sind, wird hier repräsentativ nur eine Arbeit, die sich mit diesem Phänomen bei obstruktiven Lungenerkrankungen beschäftigt [8], erwähnt, da es noch weiterer, intensiver Forschungsarbeit bedarf, um die Relevanz dieser Gen-Polymorphismen für den diagnostischen/therapeutischen Alltag abschätzen zu können.

Von besonderer Bedeutung sind allerdings bereits jetzt die Untersuchungsergebnisse von Carlberg und Haq zur individuellen Vitamin-D-Sensitivität [2]. Das von ihnen publi- 
zierte Konzept „Vitamin D Personal Response Index“ zeigt, dass die Gabe von Vitamin D in Abhängigkeit von solchen Polymorphismen zu unterschiedlichen Reaktionen des Zellstoffwechsels führt. Mit ihren Untersuchungen konnten sie nachweisen, dass etwa $50 \%$ der Testpersonen auf die Vitamin-D-Gaben regelrecht reagierten. $25 \%$ reagierten sogar ausgezeichnet. Die restlichen $25 \%$ zeigten eine deutlich eingeschränkte Reaktion. Der Umfang einer wirkungsvollen Vitamin-D-Supplementation ist daher offensichtlich nicht allein von der Höhe des gemessenen Vitamin-D-Spiegels, sondern auch vom individuellen „Vitamin D Response Index “ abhängig. Mit diesen Überlegungen bekommt nicht nur die seit Jahren geführte Diskussion über die empfehlenswerte Dosierung von Vitamin D im Rahmen der Supplementation eine neue Dimension. Vielmehr findet sich in diesem Ansatz auch eine mögliche Erklärung für „Therapieversager“ im Rahmen kontrollierter Studien - auch und gerade im Bereich des Immunsystems. Allerdings steht das von Carlberg und Haq beschriebene Verfahren noch nicht für die Routinediagnostik zur Verfügung.

\section{Hochdosis-Vitamin-D-Therapie (Coimbra-Protokoll) bei \\ Autoimmunerkrankungen}

Besonders interessant ist auch der Arbeitsansatz von CG Coimbra. Ohne die Untersuchung von Carlberg und Haq zu kennen, hat er mit seiner Arbeitsgruppe bereits vor mehr als zehn Jahren aufgrund eigener Beobachtungen damit begonnen, Patienten mit Autoimmunerkrankungen erfolgreich mit sehr hohen Dosen von Vitamin D zu behandeln, um die gewünschte Wirkung zu erzielen [5]. Allerdings handelt es sich dabei nicht - wie fälschlich immer wieder berichtet wird - um eine isolierte „Hochdosistherapie“ mit Vitamin D, sondern um eine individuelle, spezifische Dosierung im Rahmen eines ganzheitlichen Therapiekonzeptes (ähnlich dem von mir propagierten Konzept der „Spitzen-Gesundheit“). Nähere Einzelheiten zum Coimbraprotokoll und den Möglichkeiten seiner Anwendung in Deutschland finden sich auf dem Portal des CoimbraNetzwerkes (www.coimbraprotokoll.de) sowie in den Vorträgen, die im Rahmen des „1. International Workshops on Vitamin D Sensitivity and High Dose Vitamin D Therapy“ als Videos dokumentiert wurden und auf dem Webportal des Kongresses zur Verfügung stehen [9].

\section{Fazit}

Zusammenfassend lässt sich feststellen, dass Vitamin D nicht nur auf das engste mit der Regulation des Immunsystems in unserem Körper verbunden ist, sondern die gezielte Beseitigung eines Vitamin-D-Mangels außerdem zu deutlich positiven Auswirkungen bei entsprechenden Erkrankungen führt. Dabei reicht die Liste der erfolgreich behandelten Erkrankungen von einfachen über bedrohliche Infektionskrankheiten bis hin zu lästigen Allergien und schweren Autoimmunerkrankungen wie Asthma und Multiple Sklerose. Eine elementare Voraussetzung für eine erfolgreiche Behandlung ist allerdings, dass die Dosis des Sonnenhormons möglichst täglich verabreicht wird und hoch genug ist, um den Vitamin-D-Spiegel ganzjährig in einem Bereich von 40-60 ng/ml zu halten.

Damit gibt es die Perspektive, zumindest einen Teil des nebenwirkungsreichen Kortisons bei den betroffenen Patienten durch die Gabe von Vitamin D zu ersetzen. Es ist jedoch wichtig, den grundsätzlichen Aspekt, dass Autoimmunerkrankungen ein hochkomplexes Geschehen sind und Vitamin D „nur“ einer von zahlreichen Einflussfaktoren, wenn auch ein wesentlicher, ist, die alle im Rahmen eines ganzheitlichen Konzeptes berücksichtigt werden sollten, nicht aus den Augen zu verlieren [16]. Umgekehrt geht die erweiterte Bedeutung von Vitamin D deutlich über die Wirkung bei infektiösen Erkrankungen und Autoimmunerkrankungen der Atemwege hinaus und reicht von Diabetes über Demenz bis zu KHK und Krebs.

Interessenkonflikt

Der Autor erklärt, dass er Honorare von verschiedenen Herstellern von Vitamin-D-Präparaten für Vortrags- und Gutachtertätigkeiten erhalten hat.

\section{Autor}

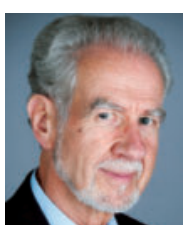

\section{Jörg Spitz}

ist Facharzt für Nuklearmedizin, Ernährungsmediziner (bfd) und Präventionsmediziner (DAPM). 2008 gründete er die „Deutsche Stiftung für Gesundheitsinformation und Prävention" und begann, Bücher zum Thema Vitamin $\mathrm{D}$ zu publizieren sowie bundesweit Vorträge zu halten. 2014 erfolgte die Gründung der „Akademie für menschliche Medizin und Spitzen-Gesundheit” sowie die jährliche Durchführung des „Kongresses für menschliche Medizin" an der Universität in Frankfurt. Seit 2015 ist er Präsident der Gesellschaft für Biologische Krebsabwehr e. V. Derzeit liegt der Schwerpunkt seiner Tätigkeit in der Erstellung von Präventionskonzepten und deren Umsetzung in der Praxis zur Förderung einer neuen Gesundheitskultur.

\section{Korrespondenzadresse}

Prof. Dr. med. Jörg Spitz

Akademie für menschliche Medizin

Krauskopfallee 27

65388 Schlangenbad

www.spitzen-gesundheit.de

E-Mail: js.amm@spitzen-praevention.de 
$\mathrm{cm} / 343$ /trends-in-der-praevalenz-von-asthma-bronchialeund-allergischer-rhinitis.pdf; Stand: 27.07.2018

[1] Alansari K, Davidson BL, Yousef Kl et al. Rapid vs. Maintenance Vitamin D Supplementation in Deficient Children with Asthma to Prevent Exacerbations. Chest 2017; 152 (3): 527-536

[2] Carlberg C, Haq A. The concept of the personal vitamin D response index. J Steroid Biochem Mol Biol 2018; 175: 12-17

[3] Chirumbolo S, Bjorklund G, Sboarina A et al. The Role of Vitamin D in the Immune System as a Pro-survival Molecule. Clinical therapeutics 2017; 39 (5): 894-916

[4] European Food Safety Authority. Vitamin D and contribution to the normal function of the immune system. Evaluation of a health claim pursuant to Article 14 of Regulation (EC) No 1924/2006 (2015). EFSA Journal 2017; 13 (7): 4182. Im Internet: www.efsa.europa.eu/de/efsajournal/pub/4182, Stand: 27.07 .2018

[5] Finamor DC, Sinigaglia-Coimbra R, Neves LC et al. A pilot study assessing the effect of prolonged administration of high daily doses of vitamin $D$ on the clinical course of vitiligo and psoriasis. Dermato-endocrinology 2013; 5 (1): 222-234

[6] Hölling H. Die „Neue Morbidität“ im Kindes- und Jugendalter. Ergebnisse der KiGGS-Studie. NIKI - Symposium Neue Volkskrankheiten im Kindes- und Jugendalter. Essen, 15.06.2016. Im Internet: www.gesund-aufwachsen.ruhr/ wp-content/uploads/2016/07/3_Homepage_VortragH\%C3\%B6lling.pdf; Stand: 27.07.2018

[7] Hollis BW, Wagner CL. The Role of the Parent Compound Vitamin D with Respect to Metabolism and Function: Why Clinical Dose Intervals can affect Clinical Outcomes. J Clin Endocrinol Metab 2013; 98 (12): 4619-4628

[8] Jolliffe DA, James WY, Hooper RL et al. Prevalence, determinants and clinical correlates of vitamin D deficiency in patients with Chronic Obstructive Pulmonary Disease in London, UK. J Steroid Biochem Mol Biol 2018; 175: 138-14

[9] Kongress für Menschliche Medizin - Update 2018. SpitzenGesundheit und Mikronährstofftherapie bei Autoimmunerkrankungen. Im Internet: https://kongress-menschlichemedizin.de/; Stand: 28.07.2018

[10] Krishnan E, Ponnusamy V, Sekar SP. Trial of vitamin D supplementation to prevent asthma exacerbation in children. Int J Res Med Sci 2017; 5 (6): 2734

[11] Martineau AR, Jolliffe DA, Hooper RL et al. Vitamin D supplementation to prevent acute respiratory tract infections: systematic review and meta-analysis of individual participant data. BMJ (Clinical research ed.) 2017; 356, i6583, DOI: 10.1136/bmj. 66583

[12] Montoya D, Inkeles MS, Liu PT et al. IL-32 is a molecular marker of a host defense network in human tuberculosis. Science translational medicine 2014; 6 (250): 250ra114, DOI: 10.1126/scitranslmed.3009546

[13] Norlin A-C, Hansen S, Wahren-Borgström E et al. Vitamin D3 Supplementation and Antibiotic Consumption - Results from a Prospective, Observational Study at an ImmuneDeficiency Unit in Sweden. PloS one 2016; 11 (9), e0163451, DOI: 10.1371/journal.pone.0163451

[14] Reichrath J, Hrsg. Vitamin D update 2012. Von der Rachitisprophylaxe zur allgemeinen Gesundheitsvorsorge. MünchenDeisenhofen: Dustri-Verlag Feistle; 2012

[15] Schmitz R. Trends in der Prävalenz von Asthma bronchiale und allergischer Rhinitis bei Erwachsenen in Deutschland 1997-99 und 2008-11 - Präsentation vom 27. März 2015. Robert Koch Institut Berlin. Im Internet: www.bfr.bund.de/
[16] Spitz J. Autoimmunerkrankungen und die 2. Evolution des Menschen. Spitzen-Gesundheit und Mikronährstofftherapie bei Autoimmunerkrankungen. Kongress für Menschliche Medizin - Update 2018, Frankfurt a. M., 14.04.2018. Im Internet: www.kongress-menschliche-medizin.de; Stand: 27.07.2018

[17] Spitz J. Vitamin D. Das Sonnenhormon für unsere Gesundheit und der Schlüssel zur Prävention. 2. erw. Aufl. Schlangenbad: Ges. für Medizinische Information und Prävention; 2009

[18] Spitz J. Vitamin-D-Mangel. Die unterschätzte Gefahr. Wien: Verlagshaus der Ärzte; 2018

[19] Spitz J, Grant WB. Krebszellen mögen keine Sonne. Vitamin D - der Schutzschild gegen Krebs, Diabetes und Herzerkrankungen. Ärztlicher Rat für Betroffene. Mit Vitamin-D-Barometer und Lebensstil-Risiko-Fragebogen. 3. Aufl. Murnau a. Staffelsee: Mankau; 2017

[20] Zhang $\mathrm{H}$, Yang $\mathrm{N}$, Wang $\mathrm{T}$ et al. Vitamin D reduces inflammatory response in asthmatic mice through HMGB1/TLR4/NFKB signaling pathway. Molecular medicine reports 2018; 17 (2): 2915-2920, DOI: 10.3892/mmr.2017.8216

Bibliografie

DOI https://doi.org/10.1055/a-0742-4576

EHK 2018; 67: 260-265

(c) MVS Medizinverlage Stuttgart GmbH \& Co. KG ISSN 0014-0082 